\title{
CORRECTION
}

\section{Correction to: Long non-coding RNA H19 promotes osteogenic differentiation of renal interstitial fibroblasts through Wnt- $\beta$-catenin pathway}

Zewu Zhu ${ }^{1} \cdot$ Yu Cui ${ }^{1}$. Fang Huang ${ }^{1} \cdot$ Huimin Zeng ${ }^{1}$. Weiping Xia ${ }^{1}$. Feng Zeng ${ }^{1}$. Cheng $\mathrm{He}^{1}$. Jinbo Chen ${ }^{1}$. Zhiyong Chen ${ }^{1} \cdot$ Hequn Chen ${ }^{1} \cdot$ Yang Li ${ }^{1}$ (i)

Published online: 7 July 2020

(c) Springer Science+Business Media, LLC, part of Springer Nature 2020

\section{Correction to: \\ Molecular and Cellular Biochemistry (2020) 470:145-155 \\ https://doi.org/10.1007/s11010-020-03753-3}

In the original article, Fig. 2c was published incorrectly. The correct version of Fig. $2 \mathrm{c}$ is provided in this correction. 
(a)

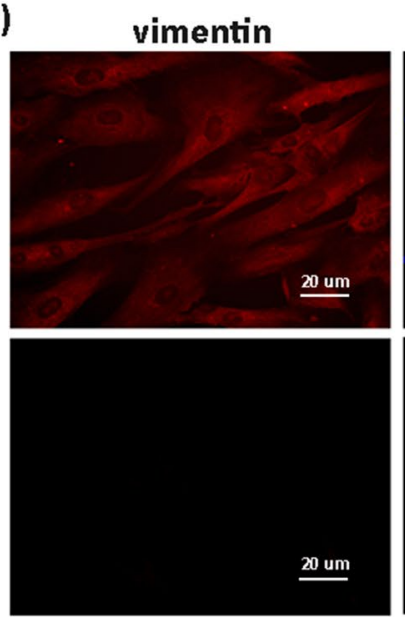

E-cadherin
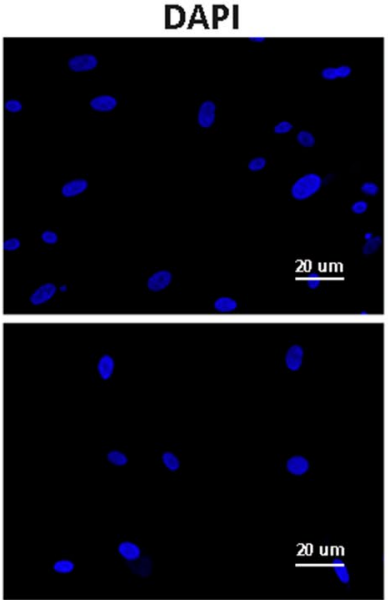

DAPI
Merge
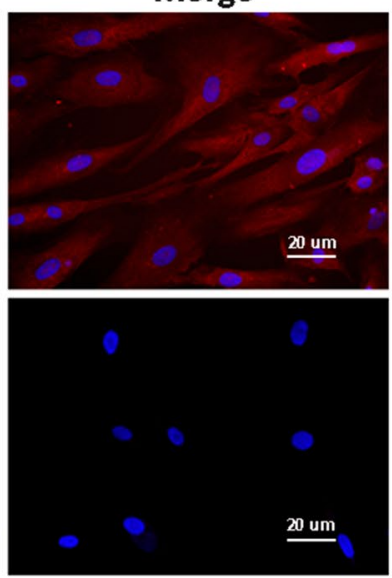

Merge (b)

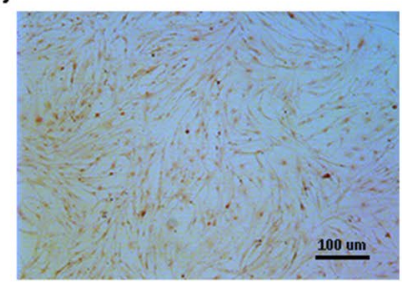

(c)

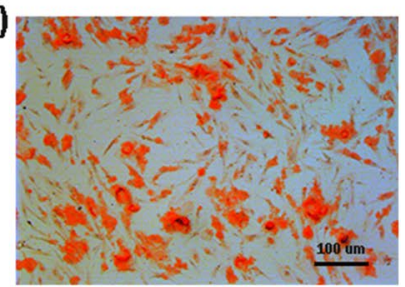

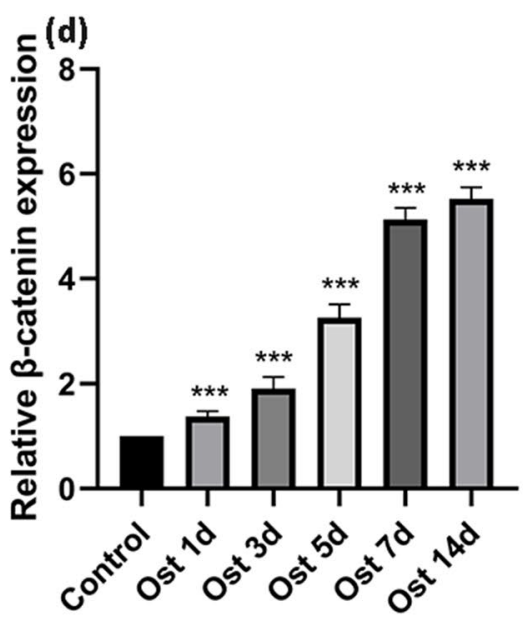

(e)
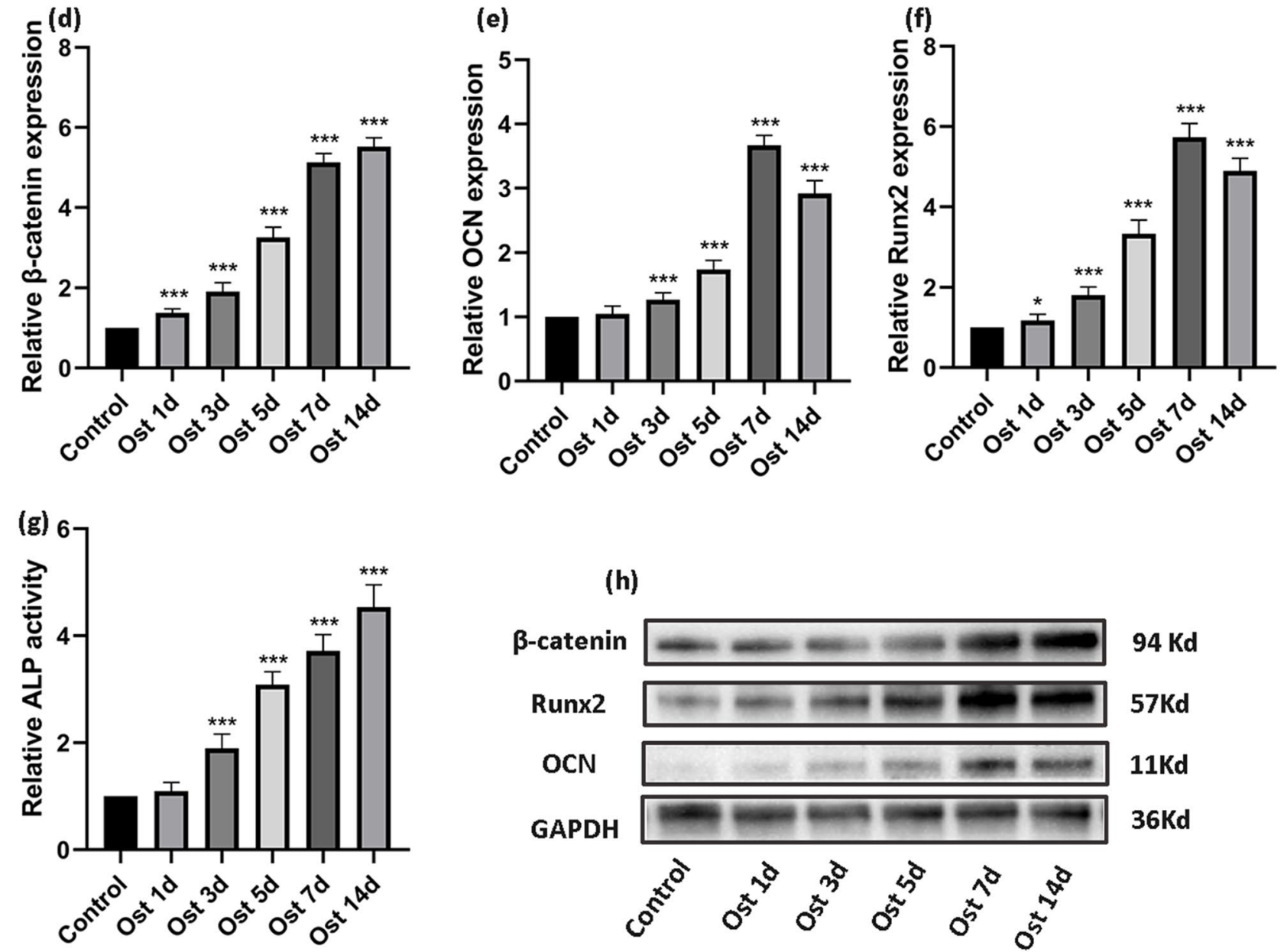

(h)

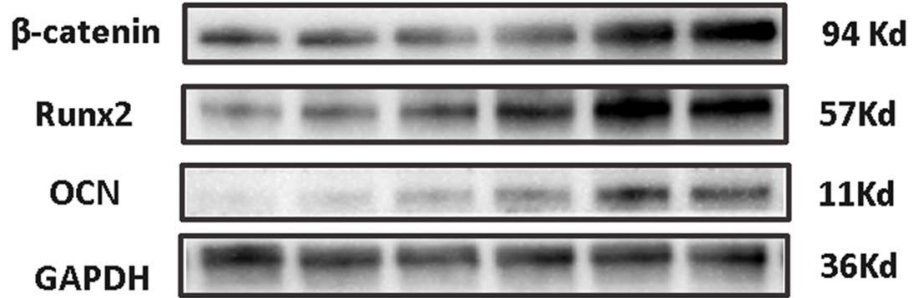

Fig. 2 Identification of primary hRIFs and osteogenic differentiation of hRIFs. a Fluorescence-labeled vimentin and E-cadherin protein were showed by fluorescence microscopy $(\times 200)$. Nuclei was stained in blue and vimentin in red, but E-cadherin was not detected. b Alizarin Red staining in hRIFs treated with normal medium for 14 days $(\times 100)$. c Alizarin Red staining in hRIFs treated with osteogenic medium for 14 days $(\times 100)$. $\mathbf{d}-\mathbf{h}$ The relative mRNA expression lev-

els of $\beta$-catenin (d), OCN (e) and Runx2 (f); the relative activity of $\operatorname{ALP}(\mathbf{g})$; and western blot analysis (h) of $\beta$-catenin, OCN and Runx 2 at $0,1,3,5,7$, and 14 days after osteogenic induction. GAPDH was used as an internal control. $* P<0.05, * * * P<0.001$ versus control. $A L P$ alkaline phosphatase, $h R I F s$ human renal interstitial fibroblasts. Runx 2 runt-related transcription factor 2, OCN osteocalcin 
Publisher's Note Springer Nature remains neutral with regard to jurisdictional claims in published maps and institutional affiliations. 